\title{
A MODE-III CRACK WITH VARIABLE SURFACE EFFECTS
}

\author{
XU WANG \\ School of Mechanical and Power Engineering, East China University of Science and Technology, Shanghai, China \\ e-mail: xuwang@ecust.edu.cn \\ Peter Schiavone \\ University of Alberta, Department of Mechanical Engineering, Edmonton, Canada \\ e-mail: p.schiavone@ualberta.ca
}

\begin{abstract}
We study the contribution of variable surface effects to the antiplane deformation of a linearly elastic material with a mode-III crack. The surface elasticity is incorporated using a modified version of the continuum based surface/interface model of Gurtin and Murdoch. In our discussion, the surface moduli are not constant but vary along the crack surfaces. Using Green's function method, the problem is reduced to a single first-order Cauchy singular integro-differential equation, which is solved numerically using Chebyshev polynomials and a collocation method. Our results indicate that the gradient of the surface shear modulus exerts a significant influence on the crack opening displacement and on the singular stress field at the crack tips.
\end{abstract}

Keywords: surface elasticity, variable surface moduli, mode-III crack, Cauchy singular integro-differential equation

\section{Introduction}

The analysis of deformation of an elastic solid incorporating a crack is critical for the understanding of failure modes and in the general stress analysis of engineering materials. Traditional modeling via the use of linear elastic fracture mechanics (LEFM) ignores the contributions of surface energies, surface tension and surface stresses. The high surface area to volume ratio present at the nanoscale dictates that any continuum-based model of deformation should incorporate the separate contribution of surface mechanics (Sharma and Ganti, 2004). Recently, the continuum-based surface/interface model proposed by Gurtin, Murdoch and co-workers (Gurtin and Murdoch, 1975; Gurtin et al., 1998) has been incorporated in the analysis of several typical crack problems (see for example, Kim et al., 2010, 2011a,b; Antipov and Schiavone, 2011; Wang, 2015; Wang and Schiavone, 2015, 2016). It was first proved by Walton (2012) and later corroborated by Kim et al. (2013) that the contribution of surface elasticity (based on the Gurtin-Murdoch model) to LEFM would, at best, reduce the classical strong square root singularity to a weaker logarithmic singularity.

The Gurtin-Murdoch surface elasticity model essentially models a material surface as a thin elastic membrane (of separate elasticity) perfectly bonded to the surrounding bulk material (see, for example, Steigmann and Ogden, 1997; Chen et al., 2007; Antipov and Schiavone, 2011; Markenscoff and Dundurs, 2014). In recent studies, the incorporation of surface elasticity into LEFM models has been confined to the simple case in which the surface moduli are constant along the crack surfaces (Kim et al., 2010, 2011a,b; Antipov and Schiavone, 2011; Wang, 2015; Wang and Schiavone, 2015, 2016).

This work aims to study, for the first time, the effects of variable surface moduli in a classical mode-III crack problem arising in the antiplane shear deformation of a linearly isotropic elastic 
solid. Specifically, the corresponding surface shear modulus is varied linearly along the upper and lower crack surfaces. By considering a distribution of screw dislocations on the crack, the problem is reduced to a single first-order Cauchy singular integro-differential equation for the unknown dislocation density which is solved numerically using Chebyshev polynomials and the collocation method. Numerical results are presented to demonstrate how the variable surface shear modulus influences the dislocation density, crack opening displacement and the singular stress field near the crack tips.

\section{Bulk and surface elasticity}

\subsection{The bulk elasticity}

In a fixed rectangular coordinate system $x_{i}(i=1,2,3)$, the equilibrium and stress-strain relations for an isotropic elastic bulk solid are well-known to be

$$
\sigma_{i j, j}=0 \quad \sigma_{i j}=2 \mu \varepsilon_{i j}+\lambda \varepsilon_{k k} \delta_{i j} \quad \varepsilon_{i j}=\frac{1}{2}\left(u_{i, j}+u_{j, i}\right)
$$

Here, $i, j, k=1,2,3$ and we sum over repeated indices; $\lambda$ and $\mu$ are the Lame constants of the bulk material; $\sigma_{i j}$ and $\varepsilon_{i j}$ are respectively the components of the stress and strain tensors in the bulk; $u_{i}$ is the $i$-th component of the displacement vector $\mathbf{u}$ and $\delta_{i j}$ is the Kronecker delta.

For the antiplane shear deformation of an isotropic elastic material, the two shear stress components $\sigma_{31}$ and $\sigma_{32}$ and the out-of-plane displacement $w=u_{3}\left(x_{1}, x_{2}\right)$ can be expressed in terms of a single analytic function $\mathrm{f}(\mathrm{z})$ of the complex variable $z=x_{1}+\mathrm{i} x_{2}$ as

$$
\sigma_{32}+\mathrm{i} \sigma_{31}=\mu f^{\prime}(z) \quad w=\operatorname{Im}\{f(z)\}
$$

\subsection{The surface elasticity}

The equilibrium conditions on the surface incorporating interface/surface elasticity can be expressed as (Gurtin and Murdoch, 1975; Gurtin et al., 1998; Ru, 2010)

$$
\begin{array}{ll}
\sigma_{\alpha j} n_{j} \underline{e}_{\alpha}+\sigma_{\alpha \beta, \beta}^{s} \underline{e}_{\alpha}=0 & \text { tangential direction } \\
{\left[\sigma_{i j} n_{i} n_{j}\right]=\sigma_{\alpha \beta}^{s} \kappa_{\alpha \beta}} & \text { normal direction }
\end{array}
$$

where $\alpha, \beta=1,3 ; \underline{e}_{\alpha}$ are the bases for the surface; $n_{i}$ are the components of the unit normal vector to the surface; [.] denotes the jump of the corresponding quantities across the surface; $\sigma_{\alpha \beta}^{s}$ are the components of the surface stress tensor and $\kappa_{\alpha \beta}$ are those of the curvature tensor of the surface. In addition, the constitutive equations on the isotropic surface are given by

$$
\sigma_{\alpha \beta}^{s}=\sigma_{0} \delta_{\alpha \beta}+2\left(\mu^{s}-\sigma_{0}\right) \varepsilon_{\alpha \beta}^{s}+\left(\lambda^{s}+\sigma_{0}\right) \varepsilon_{\gamma \gamma}^{s} \delta_{\alpha \beta}+\sigma_{0} \nabla_{s} \mathbf{u}
$$

where $\varepsilon_{\alpha \beta}^{s}$ is the surface strain tensor, $\sigma_{0}$ is the surface tension, $\lambda^{s}$ and $\mu^{s}$ are the two surface Lame parameters, $\nabla_{s}$ is the surface gradient and $\gamma=1$, 3 . In contrast to previous studies in this area, here it is assumed that $\lambda^{s}$ and $\mu^{s}$ can vary along the surface.

\section{A mode-III crack with variable surface effects}

Consider the antiplane shear deformation of a linearly elastic and homogeneous isotropic solid weakened by a finite crack $\left\{-a \leqslant x_{1} \leqslant a, x_{2}=0\right\}$. The crack surfaces are traction-free and the solid is subjected to a uniform remote shear stress $\sigma_{32}^{\infty}$. Let the upper and lower half-planes be designated the "+" and "-" sides of the crack, respectively. 
From Eq. (2.3), the boundary conditions on the crack surfaces can be written as

$$
\begin{array}{ll}
\sigma_{13,1}^{s}+\left(\sigma_{23}\right)^{+}-\left(\sigma_{23}\right)^{-}=0 & \text { on the upper crack face } \\
\sigma_{13,1}^{s}+\left(\sigma_{23}\right)^{+}-\left(\sigma_{23}\right)^{-}=0 & \text { on the lower crack face }
\end{array}
$$

where $\left(\sigma_{23}\right)^{-}$in Eq. $(3.1)_{1}$ and $\left(\sigma_{23}\right)^{+}$in Eq. $(3.1)_{2}$ are zero.

In the current setting, we have from the surface constitutive equations in Eq. (2.4) that

$$
\sigma_{13}^{s}=2\left[\mu^{s}\left(x_{1}\right)-\sigma_{0}\right] \varepsilon_{13}^{s}
$$

which indicates that the surface shear modulus is not constant but indeed variable along the upper and lower crack surfaces.

By making use of Eq. (3.2) and assuming a coherent interface $\left(\varepsilon_{\alpha \beta}^{s}=\varepsilon_{\alpha \beta}\right)$, Eqs. (3.1) are written as

$$
\begin{array}{ll}
\left(\sigma_{23}\right)^{+}=-\left[\mu^{s}\left(x_{1}\right)-\sigma_{0}\right] u_{3,11}^{+}-\frac{\mathrm{d}\left[\mu^{s}\left(x_{1}\right)-\sigma_{0}\right]}{\mathrm{d} x_{1}} u_{3,1}^{+} & \text {on the upper crack face } \\
\left(\sigma_{23}\right)^{-}=+\left[\mu^{s}\left(x_{1}\right)-\sigma_{0}\right] u_{3,11}^{-}+\frac{\mathrm{d}\left[\mu^{s}\left(x_{1}\right)-\sigma_{0}\right]}{\mathrm{d} x_{1}} u_{3,1}^{-} & \text {on the lower crack face }
\end{array}
$$

or equivalently

$$
\begin{aligned}
& \left(\sigma_{23}\right)^{+}+\left(\sigma_{23}\right)^{-}=-\left[\mu^{s}\left(x_{1}\right)-\sigma_{0}\right]\left(u_{3,11}^{+}-u_{3,11}^{-}\right)-\frac{\mathrm{d}\left[\mu^{s}\left(x_{1}\right)-\sigma_{0}\right]}{\mathrm{d} x_{1}}\left(u_{3,1}^{+}-u_{3,1}^{-}\right) \\
& \left(\sigma_{23}\right)^{+}-\left(\sigma_{23}\right)^{-}=-\left[\mu^{s}\left(x_{1}\right)-\sigma_{0}\right]\left(u_{3,11}^{+}+u_{3,11}^{-}\right)-\frac{\mathrm{d}\left[\mu^{s}\left(x_{1}\right)-\sigma_{0}\right]}{\mathrm{d} x_{1}}\left(u_{3,1}^{+}+u_{3,1}^{-}\right)
\end{aligned}
$$

The problem can be formulated by considering a distribution of line dislocations with density $b\left(x_{1}\right)$ on the crack. Consequently, the analytic function $f(z)$ can be written in the following form

$$
f(z)=\frac{1}{2 \pi} \int_{-a}^{a} b(\xi) \ln (z-\xi) \mathrm{d} \xi+\frac{\sigma_{32}^{\infty}}{\mu} z
$$

From the above expression, it follows that

$$
\begin{aligned}
& f_{+}^{\prime}\left(x_{1}\right)=-\frac{\mathrm{i} b\left(x_{1}\right)}{2}+\frac{1}{2 \pi} \int_{-a}^{a} \frac{b(\xi)}{x_{1}-\xi} \mathrm{d} \xi+\frac{\sigma_{32}^{\infty}}{\mu} \\
& f_{-}^{\prime}\left(x_{1}\right)=\frac{\mathrm{i} b\left(x_{1}\right)}{2}+\frac{1}{2 \pi} \int_{-a}^{a} \frac{b(\xi)}{x_{1}-\xi} \mathrm{d} \xi+\frac{\sigma_{32}^{\infty}}{\mu}
\end{aligned}
$$

where $-a<x_{1}<a$, The subscripts "+" and "-" here indicate limiting values as we approach the crack from the upper and lower half-planes, respectively.

It is not difficult to verify that the boundary condition in Eq. (3.4) 2 is automatically satisfied with $f(z)$ given by Eq. (3.5). On the other hand, the boundary condition in Eq. (3.4) 1 leads to the following first-order Cauchy singular integro-differential equation for the unknown density function $b\left(x_{1}\right)$

$$
-\frac{\mu}{\pi} \int_{-a}^{a} \frac{b(\xi)}{\xi-x_{1}} \mathrm{~d} \xi+2 \sigma_{32}^{\infty}=\left[\mu^{s}\left(x_{1}\right)-\sigma_{0}\right] b^{\prime}\left(x_{1}\right)+\frac{\mathrm{d}\left[\mu^{s}\left(x_{1}\right)-\sigma_{0}\right]}{\mathrm{d} x_{1}} b\left(x_{1}\right) \quad-a<x_{1}<a
$$


From Eqs. (3.6), we deduce that

$$
\Delta w=w^{+}-w^{-}=-\int_{-a}^{x_{1}} b(\xi) \mathrm{d} \xi \quad-a<x_{1}<a
$$

Consequently, for a single-valued displacement in the case of a contour surrounding the crack surface we require that

$$
\int_{-a}^{a} b(\xi) \mathrm{d} \xi=0
$$

In what follows, we assume that $\mu^{s}\left(x_{1}\right)-\sigma_{0}$ is a linear function of the coordinate $x_{1}$ and is given by

$$
\mu^{s}\left(x_{1}\right)-\sigma_{0}=\mu_{0}\left(1+\frac{k}{a} x_{1}\right) \quad-a<x_{1}<a
$$

where $\mu_{0}(>0)$ and $k(-1<k<1)$ are two constants. The constant $k$ can be considered as a parameter characterizing the gradient of the surface shear modulus $\mu^{s}\left(x_{1}\right)$ along the surfaces.

Using Eq. (3.10), Eq. (3.7) simplifies to

$$
-\frac{\mu}{\pi} \int_{-a}^{a} \frac{b(\xi)}{\xi-x_{1}} \mathrm{~d} \xi+2 \sigma_{32}^{\infty}=\mu_{0}\left(1+\frac{k}{a} x_{1}\right) b^{\prime}\left(x_{1}\right)+\frac{\mu_{0} k}{a} b\left(x_{1}\right) \quad-a<x_{1}<a
$$

Comparing Eq. (3.11) with Eq. (23) in Kim et al. (2010) reveals that a nonzero gradient parameter $k$ will result in an additional term $b\left(x_{1}\right)$ on the right-hand side of the equation. In the next Section, we present an approach based on Chebyshev polynomials and an adapted collocation method to solve Eq. (3.11) numerically together with the auxiliary condition in Eq. (3.9).

\section{Solution to the singular integro-differential equation}

We begin by setting $x=x_{1} / a$ in Eq. (3.11). For convenience, we write $b(x)=b(a x)=b\left(x_{1}\right)$. As a result, Eqs. (3.9) and (3.11) can be written in the following normalized form

$$
\begin{aligned}
& \int_{-1}^{1} \frac{\hat{b}(t)}{t-x} \mathrm{~d} t=-\pi S_{e}(1+k x) \hat{b}^{\prime}(x)-\pi S_{e} k \hat{b}(x)+2 \pi \quad-1<x<1 \\
& \int_{-1}^{1} \hat{b}(t) \mathrm{d} t=0
\end{aligned}
$$

where

$$
\hat{b}(x)=\frac{\mu b(x)}{\sigma_{32}^{\infty}} \quad S_{e}=\frac{\mu_{0}}{a \mu}
$$

Define the inverse operator $T^{-1}$ by

$$
T^{-1} \psi(x)=\frac{1}{\pi \sqrt{1-x^{2}}} \int_{-1}^{1} \psi(t) \mathrm{d} t-\frac{1}{\pi^{2} \sqrt{1-x^{2}}} \int_{-1}^{1} \frac{\sqrt{1-t^{2}} \psi(t)}{t-x} \mathrm{~d} t \quad-1<x<1
$$


and apply to Eq. (4.1) 1 to obtain

$$
\hat{b}(x)=\frac{1}{\pi \sqrt{1-x^{2}}} \int_{-1}^{1} \hat{b}(t) \mathrm{d} t-\frac{1}{\pi \sqrt{1-x^{2}}} \int_{-1}^{1} \frac{\sqrt{1-t^{2}}\left[-S_{e}(1+k t) \hat{b}^{\prime}(t)-S_{e} k \hat{b}(t)+2\right]}{t-x} \mathrm{~d} t
$$

Multiply both sides of Eq. (4.4) by $\sqrt{1-x^{2}}$ and using the condition in Eq. (4.1) $)_{2}$, we obtain

$$
\hat{b}(x) \sqrt{1-x^{2}}=-\frac{1}{\pi} \int_{-1}^{1} \frac{\sqrt{1-t^{2}}\left[-S_{e}(1+k t) \hat{b}^{\prime}(t)-S_{e} k \hat{b}(t)+2\right]}{t-x} \mathrm{~d} t
$$

We assume that the unknown function $\hat{b}(x)$ can be approximated by the following expansion

$$
\hat{b}(x)=\sum_{m=0}^{N} c_{m} T_{m}(x)
$$

where $T_{m}(x)$ represents the mth Chebyshev polynomial of the first kind.

By inserting Eq. (4.6) into Eq. (4.5), and making use of the following identities

$$
\begin{aligned}
& \frac{\mathrm{d} T_{m}(x)}{\mathrm{d} x}=m U_{m-1}(x) \quad 2 x U_{m}(x)=U_{m+1}(x)+U_{m-1}(x) \\
& \int_{-1}^{1} T_{m}(t) \mathrm{d} t=\left\{\begin{array}{cc}
\frac{1+(-1)^{m}}{1-m^{2}} & m \neq 1 \\
0 & m=1
\end{array}\right. \\
& \int_{-1}^{1} \frac{U_{m}(t) \sqrt{1-t^{2}}}{t-x} \mathrm{~d} t=-\pi T_{m+1}(x) \\
& \int_{-1}^{1} \frac{T_{m}(t) \sqrt{1-t^{2}}}{t-x} \mathrm{~d} t=\int_{-1}^{1} \frac{\left[U_{m}(t)-t U_{m-1}(t)\right] \sqrt{1-t^{2}}}{t-x} \mathrm{~d} t \\
& \quad=\int_{-1}^{1} \frac{U_{m}(t) \sqrt{1-t^{2}}}{t-x} \mathrm{~d} t-x \int_{-1}^{1} \frac{U_{m-1}(t) \sqrt{1-t^{2}}}{t-x} \mathrm{~d} t-\int_{-1}^{1} U_{m-1}(t) \sqrt{1-t^{2}} \mathrm{~d} t \\
& =-\pi T_{m+1}(x)+\pi x T_{m}(x)-\frac{\pi}{2} \delta_{m 1}-\pi x \delta_{m 0}
\end{aligned}
$$

with $U_{m}(x)$ being the $m$-th Chebyshev polynomial of the second kind, we finally arrive at

$$
\begin{aligned}
& c_{0}\left(\sqrt{1-x^{2}}+S_{e} k x\right)+\sum_{m=1}^{N} c_{m}\left[S_{e} k\left(1+\frac{m}{2}\right) T_{m+1}(x)\right. \\
& \left.+\left(\sqrt{1-x^{2}}+S_{e} m-S_{e} k x\right) T_{m}(x)+\frac{S_{e} k m}{2} T_{m-1}(x)\right]=2 x
\end{aligned}
$$

If we select the collocation points given by $x=-\cos (i \pi / N)$ for $i=1,2, \ldots, N$, Eqs. (4.8) and $(4.1)_{2}$ further reduce to the following algebraic equations 


$$
\begin{aligned}
& c_{0}\left(\sqrt{1-\left(\cos \frac{i \pi}{N}\right)^{2}}-S_{e} k \cos \frac{i \pi}{N}\right)+\sum_{m=1}^{N} c_{m}\left[(-1)^{m+1} S_{e} k\left(1+\frac{m}{2}\right) \cos \frac{(m+1) i \pi}{N}\right. \\
& \quad+(-1)^{m}\left(\sqrt{1-\left(\cos \frac{i \pi}{N}\right)^{2}}+S_{e} m+S_{e} k \cos \frac{i \pi}{N}\right) \cos \frac{m i \pi}{N} \\
& \left.\quad+(-1)^{m-1} \frac{S_{e} k m}{2} \cos \frac{(m-1) i \pi}{N}\right]=-2 \cos \frac{i \pi}{N} \quad i=1,2, \ldots, N \\
& \sum_{m=0, m \neq 1}^{N} \frac{1+(-1)^{m}}{1-m^{2}} c_{m}=0
\end{aligned}
$$

The $(N+1)$ unknowns $c_{m}, m=0,1,2, \ldots, N$ can be uniquely determined by solving the $(N+1)$ independent equations in Eqs. (4.9).

\section{Numerical results and discussion}

In Figs. 1a and 1b, we illustrate the distributions of the dislocation density $b(x)$ and the crack opening displacement $\Delta w$ for four values of the gradient parameter $k$ with $S_{e}=1$. It is observed from the two figures that: (i) $b(x)$ is no longer an odd function of $x$ and $\Delta w$ is no longer an even function of $x$ for $k \neq 0$; (ii) as $k$ increases from zero, the magnitude of $b(-1)<0$ increases considerably whereas that of $b(1)>0$ decreases only marginally; (iii) as $k$ increases from zero, $\Delta w$ increases significantly for the majority of the left portion of the crack and shrinks only marginally for a small part of the right portion of the crack. It is observed from Eq. (3.10) that the surface shear modulus for $x<0$ always decreases and that for $x>0$ always increases as $k$ increases from zero. This means that the left section of the crack becomes softer as opposed to the right portion which becomes stiffer as $k$ increases from zero. For example, when $k=0.99$, $\mu^{s}(-1)-\sigma_{0}=0.01 \mu_{0}$ and $\mu^{s}(+1)-\sigma_{0}=1.99 \mu_{0}$. In this case, the crack surface in the immediate neighbourhood of the left crack tip exhibits a minimal surface effect. Thus $-\hat{b}(-1)$ should be considerably large since it becomes infinite in the absence of any surface effect. In fact, the numerical result shows that $\hat{b}(-1) \approx-30$.
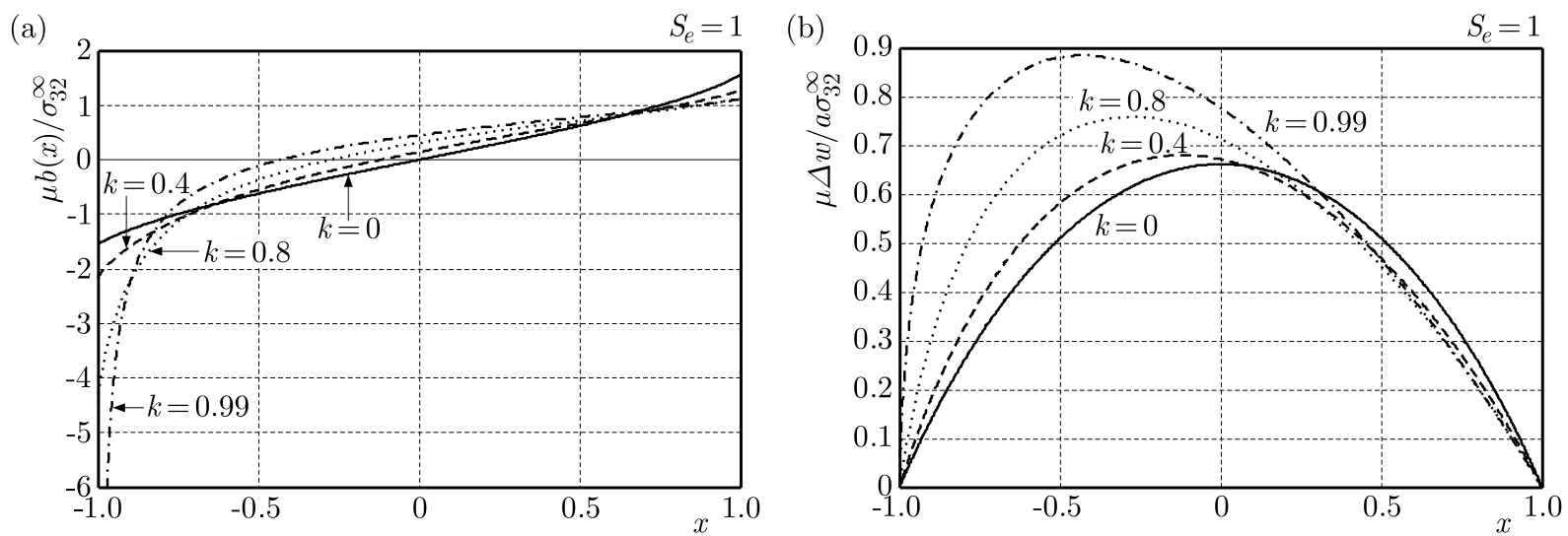

Fig. 1. The distribution of $b(x)$ (a) and $\Delta w$ (b) for four values of the gradient parameter $k=0,0.4,0.8,0.99$ with $S_{e}=1$

In Figs. 2a and 2b, we illustrate the variations of $b(x)$ and $\Delta w$ for three sets of surface parameters: $S_{e}=1, k=0.8 ; S_{e}=1.8, k=0 ; S_{e}=0.2, k=0$. The surface shear modulus at the left crack tip for $S_{e}=1, k=0.8$ is simply equal to the constant surface shear modulus in the case $S_{e}=0.2, k=0$; the surface shear modulus at the right crack tip when $S_{e}=1, k=0.8$ is 
just the constant surface shear modulus for the case $S_{e}=1.8, k=0$. It is observed from Fig. 2a that $\hat{b}(-1)=-4.4524$ for $S_{e}=1, k=0.8$ and $\hat{b}(-1)=-4.8444$ for $S_{e}=0.2, k=0$. These two values of $\hat{b}$ are clearly close to each other. In addition, $\hat{b}(1)=1.1282$ for $S_{e}=1, k=0.8$ and $\hat{b}(1)=0.9538$ for $S_{e}=1.8, k=0$. Again, these two values of $\hat{b}$ are close. From Fig. $2 \mathrm{~b}$ we see that $\Delta w$ for $S_{e}=1, k=0.8$ is greater than that for $S_{e}=1.8, k=0$ and is smaller than that for $S_{e}=0.2, k=0$. This observation is in agreement with the conclusion reached in Antipov and Schiavone (2011) that surface effects decrease the crack opening displacement. Intuitively, our observations are consistent with the physics of the problem.

(a)

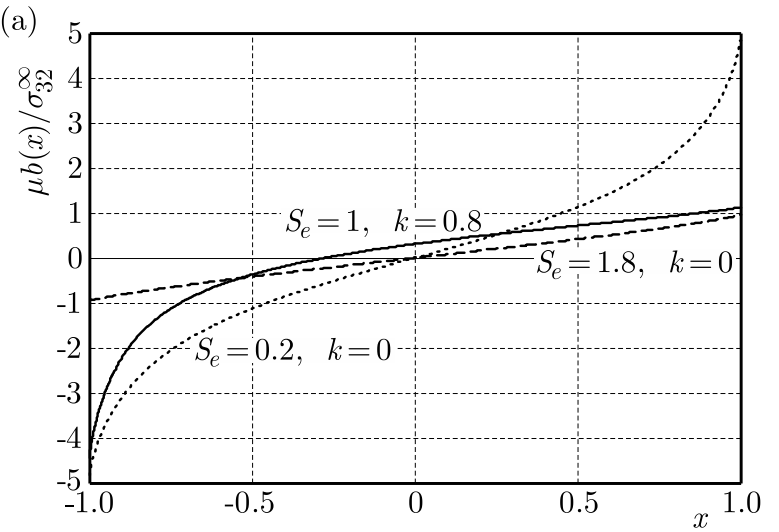

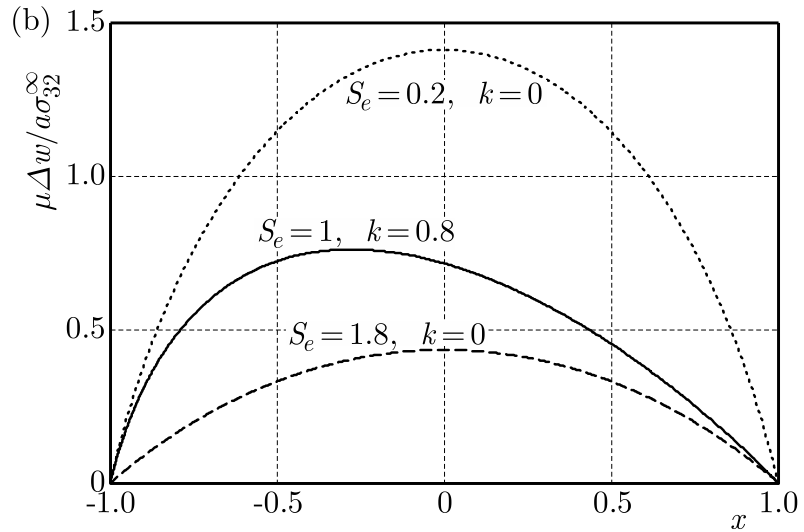

Fig. 2. The distribution of $b(x)$ (a) and $\Delta w$ (b) for three sets of the surface parameters: $S_{e}=1, k=0.8$; $S_{e}=1.8, k=0 ; S_{e}=0.2, k=0$

Once $\hat{b}(x)$ is known, the stress field can be obtained from

$$
\frac{\sigma_{32}}{\sigma_{32}^{\infty}}+\mathrm{i} \frac{\sigma_{31}}{\sigma_{32}^{\infty}}=\frac{1}{2 \pi} \int_{-1}^{1} \frac{\hat{b}(t)}{\hat{z}-t} \mathrm{~d} t+1
$$

where $\hat{z}=z / a$. Since $\hat{b}(x)$ is finite at $x= \pm 1$, the stresses exhibit a logarithmic singularity at the crack tips as follows

$$
\begin{array}{ll}
\frac{\sigma_{32}}{\sigma_{32}^{\infty}}+\mathrm{i} \frac{\sigma_{31}}{\sigma_{32}^{\infty}}=-\frac{\hat{b}(1)}{2 \pi} \ln (z-a)+O(1) & \text { as } \quad z \rightarrow a \\
\frac{\sigma_{32}}{\sigma_{32}^{\infty}}+\mathrm{i} \frac{\sigma_{31}}{\sigma_{32}^{\infty}}=\frac{\hat{b}(-1)}{2 \pi} \ln (z+a)+O(1) & \text { as } \quad z \rightarrow-a
\end{array}
$$

We illustrate in Fig. 3 the stress component $\sigma_{32}$ along the negative real axis for four values of the gradient parameter $k$ with $S_{e}=1$. As $k$ increases from zero, the material in the proximity of the left crack tip becomes softer. Consequently, as illustrated in Fig. 3, the stress increases. For any value of $k$, the stress is consistently lower than that found from the corresponding classical solution $\sigma_{32} / \sigma_{32}^{\infty}=|x| / \sqrt{x^{2}-1}$ in the absence of surface effects. In order to verify the logarithmic singularity at the crack tips, the near tip distribution of $\sigma_{32}$ along the negative real axis outside the crack is shown in Fig. 4. Seemingly, $\sigma_{32}$ is a linear function of $\ln (-x-1)$ for a fixed value of $k$. Thus the logarithmic singularity at the crack tip is verified numerically. From Fig. 4 we can also calculate the pre-factors of the logarithmic term as: -0.2738 for $k=0$; -0.3798 for $k=0.4 ;-0.7758$ for $k=0.8 ;-4.8376$ for $k=0.99$. The theoretical values from Eq. $(5.2)_{2}$ give: -0.2481 for $k=0 ;-0.3448$ for $k=0.4 ;-0.7086$ for $k=0.8 ;-4.7396$ for $k=0.99$. Clearly, the calculated pre-factors well approximate the theoretical values. 


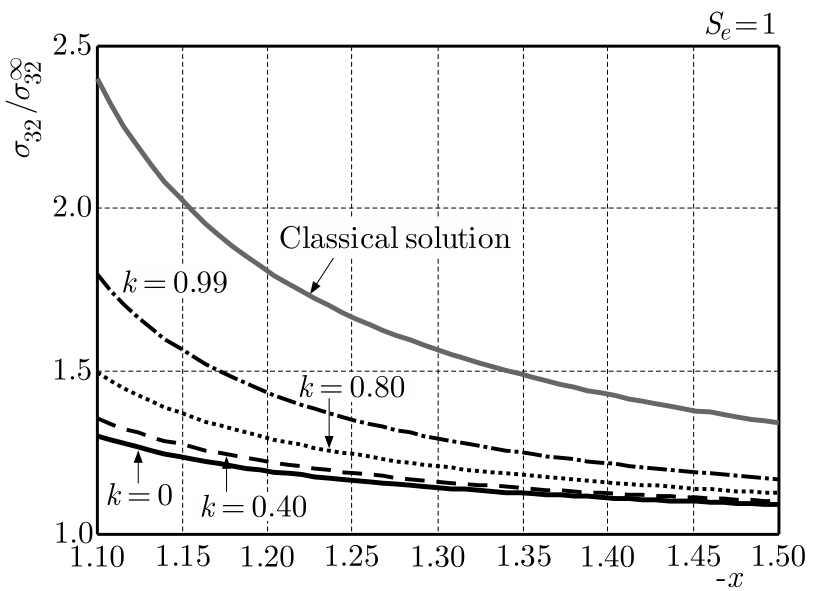

Fig. 3. $\sigma_{32}$ along the negative real axis for four values of the gradient parameter $k=0,0.4,0.8,0.99$ with $S_{e}=1$

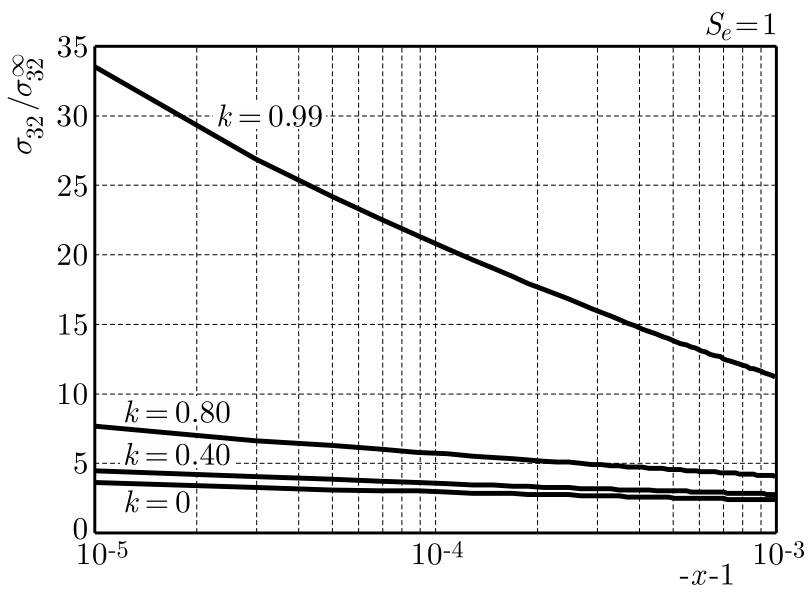

Fig. 4. The near tip distribution of $\sigma_{32}$ along the negative real axis outside the crack

\section{Conclusions}

In this paper, we utilize a modified version of the Gurtin-Murdoch model to examine the effects of variable surface shear modulus in a mode-III fracture problem arising in the antiplane shear deformation of a linearly elastic solid. The method of Green's functions is used to obtain an exact complete solution valid throughout the entire domain of interest (including at the crack tips) by reducing the problem to a Cauchy singular integro-differential equation of the first-order which is solved numerically using an adapted collocation method. Numerical results demonstrate clearly that the gradient of the surface shear modulus exerts a significant influence on the distributions of dislocation density on the crack, crack opening displacement and stress distribution near the crack tips. The numerical results also verify that the resulting analysis is correct and that the proposed collocation method is an effective tool in the analysis of crack problems in the presence of variable surface effects.

\section{Acknowledgements}

This work is supported by the National Natural Science Foundation of China (Grant No.: 11272121) and through a Discovery Grant from the Natural Sciences and Engineering Research Council of Canada (Grant \# RGPIN 155112). 


\section{References}

1. Antipov Y.A., Schiavone P., 2011, Integro-differential equation for a finite crack in a strip with surface effects, Quarterly Journal of Mechanics and Applied Mathematics, 64, 87-106

2. Chen T., Dvorak G.J., Yu C.C., 2007, Size-dependent elastic properties of unidirectional nano-composites with interface stresses, Acta Mechanica, 188, 39-54

3. Gurtin M.E., Murdoch A., 1975, A continuum theory of elastic material surfaces, Archive for Rational Mechanics and Analysis, 57, 291-323

4. Gurtin M.E., Weissmuller J., Larche F., 1998, A general theory of curved deformable interface in solids at equilibrium, Philosophical Magazine, A78, 1093-1109

5. Kim C.I., Ru C.Q., Schiavone P., 2013, A clarification of the role of crack-tip conditions in linear elasticity with surface effects, Mathematics and Mechanics of Solids, 18, 59-66

6. Kim C.I., Schiavone P., Ru C.Q., 2010, The effects of surface elasticity on an elastic solid with mode-III crack: complete solution, ASME Journal of Applied Mechanics, 77, 021011-1-021011-7

7. Kim C.I., Schiavone P., Ru C.Q., 2011a, Analysis of plane-strain crack problems (mode I and mode II) in the presence of surface elasticity, Journal of Elasticity, 104, 397-420

8. Kim C.I., Schiavone P., Ru C.Q., 2011b, Effect of surface elasticity on an interface crack in plane deformations, Proceedings of the Royal Society of London. Series A, 467, 3530-3549

9. Markenscoff X., Dundurs J., 2014, Annular inhomogeneities with eigenstrain and interphase modeling, Journal of the Mechanics and Physics of Solids, 64, 468-482

10. Ru C.Q., 2010, Simple geometrical explanation of Gurtin-Murdoch model of surface elasticity with clarification of its related versions, Science China, 53, 536-544

11. Sharma P., Ganti S., 2004, Size-dependent Eshelby's tensor for embedded nano-inclusions incorporating surface/interface energies, ASME Journal of Applied Mechanics, 71, 663-671

12. Steigmann D.J., OGden R.W., 1997, Plane deformations of elastic solids with intrinsic boundary elasticity, Proceedings of the Royal Society of London. Series A, 453, 853-877

13. Walton J.R., 2012, A note on fracture models incorporating surface elasticity, Journal of Elasticity, 109, 95-102

14. WANG X., 2015, A mode III arc shaped crack with surface elasticity, Zeitschrift für Angewandte Mathematik und Physik, 66, 1987-2000

15. Wang X., Schiavone P., 2015, A mode III interface crack with surface strain gradient elasticity, Journal of Integral Equations and Applications, 28, 123-148

16. Wang X., Schiavone P., 2016, Bridged cracks of mode III with surface elasticity, Mechanics of Materials, 95, 125-135 